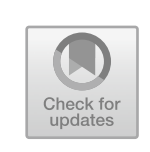

\title{
Defining Ideas of Quantum Physics
}

Quantum physics was born to describe the curious and common-sense denying micro-universe within the atom. Today, physicists tell us that it is actually the most accurate way to describe everything that exists, including ourselves. They tell us that we live in "a quantum world." This world is made of energy, not "bits of matter," and everything and everyone that exists in our world is actually an evolving pattern of dynamic energy. You and I are patterns of dynamic energy, and our companies are dynamic energy systems. The quantum world is also made of relationships, and everything and everyone is in relationship with, is "entangled" with, everything and everyone else. This is "quantum holism." The quantum world is indeterminate, and thus unpredictable, creatively "feeling" its way into the future through self-organization. It is a world where questions are more important than answers, and we people who ask those questions "co-authors of reality."

These, and other key defining elements of the quantum world are spelled out in greater detail here, and contrasted with the defining elements of the earlier, Newtonian physics and Newtonian paradigm. These basic quantum concepts and principles are the necessary building blocks for understanding Quantum Management and its place in the wider quantum paradigm, and thus readers may need to refer back to them while going through the book. I believe this is basic science that 
every twenty-first-century leader should know, especially business leaders, and I will be spelling out the relevance and wider leadership implications of these quantum concepts throughout the book.

\section{QUANTUM INDETERMINISM}

The most powerfully revolutionary feature of Newtonian physics was the certainty it offered. If everything in the physical universe was controlled by three inviolable, mechanistic laws, then by using those laws we could, infallibly, predict the future trajectory of any moving particle or unfolding event. Furthermore, if we knew how to employ those laws of motion ourselves, we could control the outcome of events. It is little wonder that, as the decades and centuries passed, this Newtonian determinism became an attractive model for everything from the behavior of individuals and societies to that of markets and companies. But in the rapidly changing, complex, and apparently chaotic world of the late twentieth and then twenty-first centuries, relying on the deterministic certainty of behavior and events to guide decision-making has become a hindrance rather than a help. Decisions based on "the best laid plans" just keep blowing up in our face.

I have said that the new quantum paradigm turns everything we thought we knew upside down and on its head. And one of the many revolutionary discoveries of quantum physics is that there is no such thing as certainty. When an electron makes a quantum leap from one energy orbit to another inside an atomic system, there is no predicting when that leap will take place, or how big the leap will be. And if the nucleus of a radioactive atom emits a particle, there is no telling when this decay event will be. There don't seem to be any laws controlling events in the radically contingent quantum universe. Things just happen as they happen. We don't see them coming, and we have no idea where they are going.

Quantum events are probabilistic. Some are more likely than others to happen. Given a large enough number of events, we can predict certain patterns of outcome. Schrodinger's wave equation (see below) describes all possible outcomes of a quantum event, now and in the future, but this is only a set of bookmaker's odds. We can rarely predict anything useful about the future behavior of a single quantum event. Newton's vision of a universal clockwork machine gives way in quantum physics to a universal roulette wheel, or a game of dice. This outraged Einstein, who protested, "God does not play dice with the universe!", and he and others tried 
to argue that the uncertainty was a result of our limited knowledge, not a feature of reality itself. But countless experiments proved him wrong. Radical contingency really is a fundamental feature of our world.

Within the categories and values of the exhausted Newtonian paradigm, quantum indeterminacy appears as a threat or a negation of everything science has offered us, but it is the very foundation of what the new quantum paradigm sees as positive. It is precisely because the outcome of quantum events is indeterminate that its uncertainty underpins the potentiality, or the "what might be," of an evolving system or situation. It holds out the possibility of unlimited creativity and innovation. Because the many variables of a quantum system begin as unfixed or ambiguous, the system is free to evolve in co-creative dialogue with other systems, with its environment, or with conditions prevailing in human affairs. It frees our hands to be spontaneous, or flexible. Thus, just as knowing the iron laws of Newton's physics allowed leaders of the Machine Age to thrive on certainty, understanding, and learning to work with the principles of quantum physics can help leaders of our twenty-first-century Digital, or Quantum, Age, thrive on uncertainty.

\section{HOLISM}

Like the word "quantum" itself, being "holistic" has acquired so many uses in the popular vocabulary that it has lost most of its original, and really powerful meaning. But just as any proper understanding of the broad, new quantum paradigm requires that we know quanta are packets of energy, and a single quantum is the smallest amount of energy required to make anything happen in our universe, which is itself made of energy. A useful understanding of quantum holism is necessary to living, acting, and leading effectively in what is now the zero distance, Quantum Age.

Newtonian physics, and all the social, business, and political models inspired by it, is atomistic. Taking atoms, which are separate and isolated, as the basic building blocks of the universe, the atomistic thinker believes that any whole can be broken down and analyzed into separate parts (its "atoms") and the forces acting between them. True knowledge about what anything is, or how it works, is thus achieved by isolating the thing and then reducing it to its constituent parts. The whole thing is always thought to be just the sum of its parts. The human body is a brain, plus lungs, plus a heart, plus kidneys, etc., and a human being is a mind, plus a body. Thus, a top doctor becomes a brain specialist, a lung specialist, a 
kidney specialist, etc., and a patient suffering an illness is referred to the relevant specialist. Until very recently, and now only with a few recognized exceptions, a patient suffering from stress is sent to a psychiatrist or psychologist, but another suffering with ulcers is sent to a gastrointestinal specialist. Western medicine has no whole-system understanding of mind/body complex.

By contrast, quantum physics, which holds that quanta of energy are the basic building blocks of the universe, teaches us there is no such thing as separation, that every quantum of energy in the universe, and everything made of quanta, is "entangled" with everything else. Nothing really is anything identifiable or knowable except in relation to the whole of the system of relationships with which it is entangled. This is because quantum "parts" acquire many of the properties they have through those relationships (see Contextualism, below). And they have the potential to have different properties if they then find themselves in a different relationship. Furthermore, each quantum part has the potential to have further properties when it is in relationship to other parts. The very combination of parts in any system of relationships gives that combination more, or different, characteristics or properties than originally possessed by the parts. A quantum whole is always larger than the sum of its parts. This is the quality known as quantum emergence, and it makes the constant creation of something new a feature of the quantum world. Life is an emergent property of certain inorganic chemical elements combining in a given relationship, a human being is an emergent phenomenon resulting from a relationship of body parts, and consciousness itself is thought to be an emergent phenomenon of brain processes, quite likely in relationship to those of the body.

Thus quantum holism maintains that the whole is more primary than any parts of which it is constituted. A holist always looks at things or systems in aggregate, and knows that any true understanding of them rests on understanding the relationships between them. This has far reaching implications for how we understand teams and organizations.

\section{Both/And: Waves ANd Particles}

One of the big challenges to Newtonian physics was its inability to say whether light is a stream of particle-like photons, or a series of wavelike photon patterns. Some experiments indicated one thing, but then 
other experiments indicated the opposite. William Bragg, a Noble prizewinning British physicist whose distinguished career spanned the late nineteenth and early twentieth centuries, expressed this confusion when he said, "Light seems to behave like a wave on Mondays, Wednesdays, and Fridays, and like a particle on Tuesdays, Thursdays, and Saturdays." Quantum physics answered the conundrum effectively by saying, "Every day is a Sunday." Light is both wave-like and particle-like, and to different degrees, always at the same time. This is called wave/particle duality. And it is true of every quantum entity: all matter in the universe can always behave as though it has both wave-like and particle-like properties.

The both/and nature of quantum reality also extends to positions in space and time. Where both Aristotelian and Newtonian logic insist that a particle is either here or there, that it has interacted with another particle either now or then, experiments in the quantum laboratory have proven that particles and their interactions are spread out all across space and time. Particles can be both here and there, interactive events can be both now and then. And this same quantum logic applies to our statements, decisions, and emotions. A statement can be both true and false at the same time, a decision both bad and good at the same time. And we all know that we can both love and hate a life partner or a friend at the same time. Those who speak the Mandarin language also know that a word or a phrase can always mean this and that (and many other things besides!), and any Indian will tell you, "Yes, we drive on the left, and we also drive on the right." We will see later that the both/and nature of quantum logic raises important questions about the preferred nature of corporate planning and decision-making, and makes us think twice about "one best way" or "one best product or service."

As physicists now tell us we live in a quantum world, we know that all things have quantum properties. Thus wave/particle duality is literally true to a very small extent even of large entities like ourselves, or our companies. But if we extend the both/and nature of waves and particles as a principle of how we think, then it can also serve as a powerful metaphor for better understanding the dynamics of our human relationships and the nature and potential of our companies or other organizations. In some circumstances, it can be best to think of these as having the characteristics of overlapping waves, co-mingling and co-creating with whomever or whatever is around us. Yet in other circumstances or ways, it can be best to stand out, or stand back, and behave as though we are particles that have boundaries and can act as independent individuals. 


\section{QUANTUM Non-LOCALITY}

However, just to make this new understanding of wave/particle duality still more challenging, even particles are not entirely separate in the quantum world. The fact that every particle also has a wave nature that can be entangled with the wave nature of other particles, means that even apparently separate particles can be nonlocally correlated, their movements or characteristics acting in mirror-like synchrony with those of others.

If a light source emits two, different photons in opposite directions, and a scientist then measures each of them when they have traveled to the far ends of a very long room, he finds that the polarities of the two photons are synchronized, the polarity of one is always opposite to the polarity of the other. No force or signal has passed between these photons to inform them about the state of each other, there is no hidden string or spring connecting them, and no one has informed them beforehand which polarity to adopt. The photons just seem to "know" what each other are doing. When this "quantum non-locality" was first predicted, Einstein was outraged, called it "ghostly and absurd," and protested it would mean that photons have telepathic powers. It was indeed the feature of quantum physics that he hated most, even though he had been one of those who first discovered the new science. But in the late 1970s, experiments proved the existence of non-local correlation beyond any doubt, and it was soon explained that, though the photons as particles were separate when measured across a distance, as waves they were entangled, and no amount of "distance" had any meaning. And this would apply even if the photons had traveled to opposite sides of the universe before being measured.

Today, quantum non-local correlations are known to be part of our everyday world. Non-local correlations between electrons in their eyes now explain the once great mystery of how birds maintain their flocking patterns during migration, and these correlations also play a key role in plant photosynthesis. They may well explain many forms of human communication like crowd behavior, the beautiful, dancer-like synchrony of football players' movements during a match, or even things anecdotally reported such as telepathy and precognition. Being such a critical defining feature of quantum systems, including much of the quantum technology upon which twenty-first-century industries rely, it is quantum non-locality that perhaps stands out as the flagship on our voyage into today's zero distance world where having something local is no longer an issue. 


\section{Contextuality: Relationships Matter!}

When we learned middle school science, we were taught that the first step of the Scientific Method is that you isolate the thing you want to study from its environment. Built into this instruction is the assumption that a failure to isolate will confuse or contaminate our knowledge of the thing itself. Things are only purely or truly themselves when separated from other things. But quantum science stands this whole methodology on its head. It tells us there is no such thing as separation, that everything is in relation to, is "entangled with," everything else, and that therefore the very concept of isolation is wrong-headed. If a quantum physicist wants to know the nature and properties of any particle, or any element of a system, he knows that he must know what surroundings it is in, what it is in relationship to, what is its role in the whole system. This is the principle of quantum contextualism: things never "are just what they are," but rather, things "are what they are in relationship with." The nature of a thing, the identity, of a thing is determined by its context, its relationships.

This contextual nature of quantum reality is made clear in its boldest form in the outcome of what is probably the most famous experiment in quantum physics, "the two-slit experiment." This is the experiment that proved the dual wave/particle nature of light. In the laboratory, a beam of photons is directed toward an opaque barrier, and this barrier has two slits, or "gates," either one of which the scientist can open or close. If just one slit is opened, the photons pass through as a stream of particles and go "click, click, click" on a particle detector (a photomultiplier tube) that has been placed behind the barrier. But if the scientist opens both slits, the photons pass through as a series of waves and make an interference pattern on a wave detector (a white screen that has also been set up on the other side of the barrier.) Light is both wave-like and particle-like at the quantum level of reality, but it manifests as either wave-like or particle-like at our everyday level of visible reality, depending upon the context-i.e. whether its environment is a single open slit or two open slits.

This contextual, or relationship-dependent nature of quantum things and events also turns on its head another sacred truth of ancient Greek philosophy and Newtonian physics, causality, or The Law of Cause and Effect. When we want to know why something has happened, we are accustomed to seeking its cause. We ask, "What caused this argument?" 
"What caused my company to fail?" "What caused the financial recession?" Built into every "Why?" that we ask is the assumption that a cause lies behind the event or the effect we are questioning. But the discoveries of quantum physics tell us that such causal thinking is wrong-headed. There are no causes, quantum scientists tell us, there are only relationships.

Indeed, according to quantum physics, reality itself exists because there are relationships. Relationships, we are told, make reality. Of the two basic types of particles of which everything in the universe is made, bosons and fermions, bosons are themselves particles of relationship. The Four Fundamental Forces that bind the universe and everything in it together-gravity, electromagnetism, the strong and weak nuclear forces, are made of bosons. And the things they bind together are all made of fermions. As Plato said, wherever we have two or more "things," there will also always be present the relationship between them.

So a basic principle of the quantum paradigm is: Don't ask what cause is, or should be, present here, but ask instead, what is the relationship that exists, or needs to exist, in this situation? To achieve what we want or to improve our conditions, we don't add or alter causes, but rather we add or alter relationships. It is obvious to most of us that the quality of our personal lives is dependent upon the nature and quality of our relationships, and the health of a community or society depends upon the quality and extent of its network of relationships. And when we discuss the nature of a quantum company or organization, we will see that all human organizations are, in large part, systems of relationships, indeed systems of relationships within larger systems of relationships. Just as it is true in physics that relationships make reality itself, it is the quality of its internal and external relationships that makes a company a good or bad company, and that determines whether it will be a successful or a failing company. Thus systems thinking will be seen as a critical skill of quantum leadership.

\section{A Universe Made of Energy}

Like the Greek philosopher Democritus, Newton asserted that everything is made of atoms, and the Newtonian atom was visualized as a tiny, solid, material object very much like a miniature billiard ball, its boundaries hard and impenetrable. Newton said that atoms were the ultimate building blocks of the universe and everything in it, and as the Newtonian scientific paradigm became the general paradigm for all Western thinking, 
Newton's atoms became the metaphorical building blocks and structural models for things as diverse as plants and animals, the human psyche, human relationships, societies, markets, and companies. But the discoveries of quantum physics and quantum field theory have blown atomism out of the water.

According to quantum science, the universe and everything in it, is in fact made of energy. The very source and foundation of the quantum universe, the Quantum Vacuum, is itself a still field of energy, like a very calm, mirror-like sea without waves, the field of all fields. And then all existing things-elementary particles of "matter," stars, planets, everything living and dead on our Earth including ourselves, our relationships, our companies, etc.- - are oscillating "waves" of energy on some field, which is itself a field upon this background "sea." Every existing thing is a dynamic pattern of energy "waving" upon the Quantum Vacuum or on one of the many other energy fields that emerge from it. "Matter" itself, including what we take to be our own very material bodies, is in fact just extremely dense, highly compressed, patterns of energy. The universe is not made of material atoms, as Newton said, but rather of "quanta" of energy. A "quantum" of energy, the smallest packet of energy that can make anything happen or exist, is in fact the irreducible building block out of which everything is made. Atoms themselves are made of quanta.

As we go through the remaining chapters of this book, the revolutionary fact that everything and everyone is in fact a dynamic wave pattern of energy oscillating on the common, universally shared, background energy sea of the Quantum Vacuum will figure largely in our understanding of the new quantum paradigm, and how this changes everything we have ever thought about anything. This includes the promised new understanding of what it means to be human, and how we human beings live, work, lead, and manage our companies. Indeed, how we understand what a company is.

\section{A Universe of “Real” Potentiality}

In normal discourse, when we speak of a person's or a system's “potential," we mean something that lies in the future, a promise of some ability or use that is yet to show itself, and we might say, "This student is not performing up to his potential," or "There is vast potential in our workforce." Both statements imply there is a lingering ability or resource yet to be realized or discovered. "Quantum potentiality" does have some of 
this same meaning, but it refers still more powerfully to properties of a quantum system that are present in the here and now, properties built into the system's identity, and thus to properties that must be considered as active players in any given situation involving the system. A quantum system's potentiality is an explanatory factor in how a system behaves, the affects that it has on other systems, or its role in an unfolding situation. Thus, in quantum physics, potentiality is as "real" as what we call reality.

The quantum physicist David Bohm described two orders, or levels, of reality that he called the Implicate Order and the Explicate Order. As their names suggest, the Implicate Order is hidden, but always implied, and the Explicate Order is what is actually seen, what is manifesting now, i.e., what is explicit. In Bohm's model, The Implicate Order is the realm of quantum potentiality, where all things are possible, and the Explicate Order is the realm of everyday perception and experience, where only one of an infinite array of potentialities rises to the level of what we normally call reality. Thus, like the Quantum Vacuum itself, The Implicate Order is a sea of possibilities and the Explicate Order the waves that stand out upon that sea. And just as the sea and the waves upon it are one continuous medium, so the Implicate potentialities of a "thing" are within, are the ground substance of, its Explicate manifestation.

In the example of Schrodinger's alive/dead cat that we read about in the Introduction to this book, in the Implicate realm (inside his unopened box) the cat is both alive and dead at the same time, just as the quantum nature of light (a beam of photons) is wave-like and particle-like at the same time. But when we view the cat in the Explicate realm by opening his box, he is either alive or dead, just as the light becomes either a stream of particles or a series of waves when it enters the Explicate realm by passing through one or two open slits in the opaque barrier.

In fact, there are not just two distinct possible potentialities for either Schrodinger's cat or the beam of light while they reside in the Implicate realm of being, nor for any other quantum "thing," but rather each has an almost infinite array of possible outcomes for how it will manifest in the Explicate realm. Everything existing in the Implicate Order exists as a wave of superimposed, often contradictory, potentialities, each displaying a range of probable outcomes, and this "probability wave," or "wave function" is described in the famous "Schrodinger's wave equation." Everything in the universe, including you and me and even any company that we might manage, has a wave function. Each of us, at one 
level of our being, is a wave of potentialities waiting to manifest as "real" in one way or another.

Until the twenty-first century, most quantum physicists believed that only one potentiality could ever manifest, that for some unknown reason the Schrodinger wave function "collapsed" as many potentialities entered the Explicate Order and became one actuality. And this apparent "collapse of the wave function" was the great unsolved mystery, perhaps, some thought, even the tell-tale incompleteness or wrongness, of quantum theory itself. But today, the vast majority of physicists accept that the wave function doesn't actually collapse after all. Many follow the Many Worlds view that all potentialities manifest as real outcomes, but that each does so "in a different world," that reality itself splits into different worlds every time a wave function is confronted with an array of different probable outcomes. Thus Schrodinger's cat is alive in one world and dead in another.

Other physicists, myself included, believe the Many Worlds view to be a metaphysical nonsense. I prefer David Bohm's view that the wave function spreads out into one, fully realized, visible outcome made most probable by its interaction with the surrounding environment, and into an accompanying array of other, partially realized, outcomes, all remaining in an interactive dialogue with each other and thus all having an active influence on the situation. It is in this Bohmian view that potentiality is always a hidden side of actuality. What we are, or are doing, now is always entangled with what else we can be, or might be doing. I will be following this Bohmian view throughout the book, and it plays a significant role in how I will describe the nature and dynamics of a quantum self, a quantum organization, and a quantum society.

\section{Heisenberg's Uncertainty Principle: Questions Create Answers}

Both philosophers and scientists wonder at times how much we can ever know about fundamental physical reality. They ask whether ambiguity and uncertainty are inherent features of the real world, or is our knowledge about reality always incomplete, for some reason always necessarily limited? Heisenberg's Uncertainty Principle, the most famous principle in quantum physics, addresses exactly these questions. And it is important that leaders know about it because it has wide implications for how we understand and manage our organizations. 
Much like the new science of behavioral economics says about the market, quantum reality is a strange, uncertain, shadowy realm. The more we try to pin it down and make rational models of it, the more it eludes us. The Uncertainty Principle tells it must always be so; because quantum reality itself is indeterminate, we must always content ourselves with partial truth and ambiguity when trying to know it.

Newton's physics said that a particle always has both position and momentum. It should always be somewhere, and always traveling at a definite speed. But due to the strangeness of the quantum world, we can never know both at the same time. If we measure, or focus on position, the momentum becomes fuzzy and indeterminate; if instead we measure momentum, then the position eludes us. It is the same with all the other complementary pairs of properties that particles can possess: waves and particles, energy and time, or continuity and discontinuity. Fixing our attention on any one property of any pair always makes the other property become fuzzy and indeterminate.

David Bohm later pointed out that it is the same with our conscious attention in human situations. If we conduct an interview with a job applicant, for instance, we can ask them a series of detailed, factual questions and compile a written report of their skills and past work experience, but when the applicant leaves the room we won't have gained much sense of them as a personality. Or, if instead, we conduct the interview as an informal chat intended to gauge what kind of person they are, they will then leave the room with our knowing few actual facts about them. In our organizations, we often find we must choose between laying down rigid rules and tight structure, or allowing things to creatively self-organize. Tight structure gives us control, but it loses us the benefits of innovation.

The other, and perhaps most important, lesson leaders can take away from Heisenberg's Uncertainty Principle is that the questions we ask give us the answers that we get. If we ask different questions, we get different answers. More importantly, we can see that asking good questions is like dropping a bucket into a well of potential answers, and then bringing some of those back to the surface with our rope. But given the co-creative relationship in quantum physics between the observer and the observed, between the question and its answer, we can see that the art of asking good questions is more like dropping that bucket into the sea of quantum potentiality and then bringing up a bucketful of new reality. Questions don't just fetch answers; questions create answers. And for this reason, we will see later that a quantum organization is an organization that fosters and encourages questions - and questioning! 


\section{A Participatory Universe}

One of the reasons for so many people feeling powerless today is that we feel we have little or no influence or control over how events in this world unfold. Even in democracies, citizens often have the sense that their vote doesn't really count, that either the "system is rigged" or the politicians they elect won't do what they have promised anyway. In Newton's physics, there were grounds for this sense of powerlessness on a cosmic scale. There simply was no place in his mechanistic universe for human beings, our consciousness, our intentions, our hopes, or dreams. Conscious creatures like ourselves were accidental bystanders in a universe wholly governed by the iron, deterministic laws of the clockwork universe. We could stand back and observe a world "out there," but our observations had not the slightest effect on what we observed. The material world had its own reality, and obeyed its own laws, quite apart from what observers see or do, or indeed whether there even are any observers.

The discoveries of quantum physics changed all this, putting human consciousness and intention squarely at the center, not only of how the universe works, but even of what comes to exist in it. We have already seen that the way a scientist sets up his experimental apparatus determines the result his experiment will produce. The quantum observer stands inside his or her observations, and those observations themselves play an active role in bringing about the very reality they look at. According to quantum science, the observer and what he or she observes are one, co-creative, partnership in what gets observed. In a sense not yet fully understood, the quantum observer makes the world of his or her observations.

The unobserved world of the Implicate Order is a plethora of possibilities. We have already seen that Schrodinger's wave equation describes an infinite array of possible states into which any quantum entity may emerge. All this possibility becomes actuality only when the wave function comes into relationship with something in the environment that then makes one of its potentialities stand out as an existing thing in the Explicate Order - the world of daily reality. And the mere act of measurement-making an experimental observation, asking an experimental question, is one thing that teases a quantum entity into existence. Further, the kind of measurement that is made determines which kind of actuality will be plucked from the sea of infinite possibility. In other words, the observer sees what the observer looks for. As we learned from 
Heisenberg's Uncertainty Principle, the questions we ask give us the answers that we get.

Thus, quantum physics tells us that we live in a participatory universe in which our questions, our projects, our experiments, our decisions, even our character, make the world we live in. This has enormous implications for our role in the world and our responsibility for the world, and we will explore these more fully in later chapters.

Open Access This chapter is licensed under the terms of the Creative Commons Attribution-NonCommercial-NoDerivatives 4.0 International License (http:// creativecommons.org/licenses/by-nc-nd/4.0/), which permits any noncommercial use, sharing, distribution and reproduction in any medium or format, as long as you give appropriate credit to the original author(s) and the source, provide a link to the Creative Commons license and indicate if you modified the licensed material. You do not have permission under this license to share adapted material derived from this chapter or parts of it.

The images or other third party material in this chapter are included in the chapter's Creative Commons license, unless indicated otherwise in a credit line to the material. If material is not included in the chapter's Creative Commons license and your intended use is not permitted by statutory regulation or exceeds the permitted use, you will need to obtain permission directly from the copyright holder.

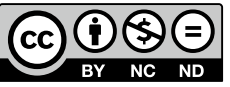

